\title{
The Choroidal Circulation and Retinal Metabolism-An Overview
}

\author{
WALLACE S. FOULDS, CBE \\ Glasgow
}

In most species, including man, the outer retina is totally dependent on the blood circulation in the choroid for its metabolic needs. In some species, such as the rabbit which lacks retinal vessels, the choroid supports the metabolic requirements of the whole thickness of the retina. This situation is also true of the macular retina in primates including man. There are obvious visual benefits in having a retina of reduced vascularity, not the least of which is the unimpeded transmission of the visual image to the receptor cells of the retina. A disadvantage is the relative remoteness of the outer retina from its source of nourishment and the interpolation of various structures between the choroid and the retina, any of which may become abnormal and interfere with the supply of nutriments to the retina or with the elimination of the waste products of retinal metabolism.

An immediately obvious peculiarity of the choroidal circulation is the fact that it appears to be greatly in excess of even the high metabolic requirements of the retina. The extraction of metabolites from the choroidal circulation is incomplete and blood in the vortex veins leaves the eye highly oxgenated with only a small proportion of its haemoglobin in the reduced form. There is an analogy in the kidney for blood in the renal veins like that in the vortex veins is bright red and highly oxygenated. Blood flow in the choroid has been measured by many workers using a variety of techniques. Anders Bill in Sweden has been active in this area for many years using direct measurement from a divided vortex vein or embolisation by radioactive spheres. ${ }^{1} \mathrm{We}$, in Glasgow, using a radioactive Krypton washout technique showed that the choridal blood flow is approximately ten times cerebral blood flow when considered on the basis of blood flow per unit mass of tissue. ${ }^{2}$

The high blood flow in the choroid is at least in part related to low resistance in the choroidal capillaries which are very wide bore as compared, for example, with retinal capillaries (Fig. 1). Additionally there is a very generous arterial blood supply via the ciliary arteries which arise directly from the ophthalmic artery. The work of Hayreh on the segmental supply of blood to the choroid ${ }^{3}$ has had a profound influence on our understanding of choroidal vascular insufficiency. The question of homeostasis in the choroidal circulation is still debatable. Wilson and Strang ${ }^{4}$ in Glasgow

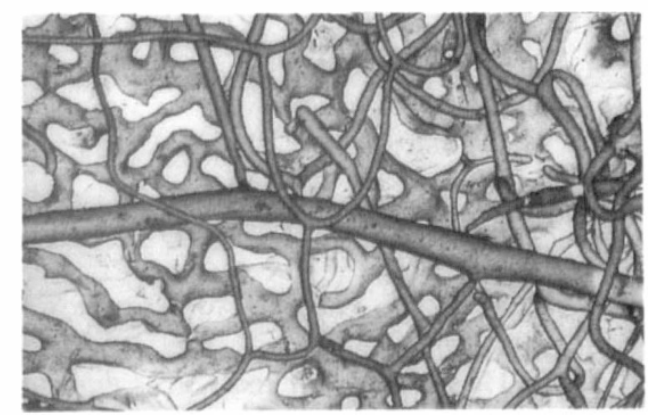

Fig. 1. Scanning electronmicroscopy of a resin cast specimen of the retina and choroid to show the considerable disparity in size which exists between retinal capillaries and the underlying chorio-capillaris.

From: Tennent Institute of Ophthalmology, University of Glasgow, Western Infirmary, 38 Church Street, Glasgow G11 6NT.

Correspondence to: Wallace S Foulds, CBE, Ross Hall AMI Hospital, 221 Crookston Road, Glasgow. 


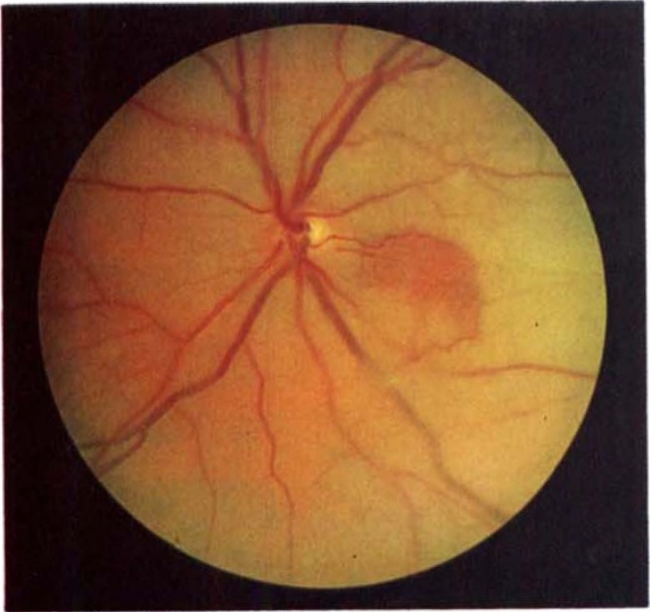

Fig. 2 a.

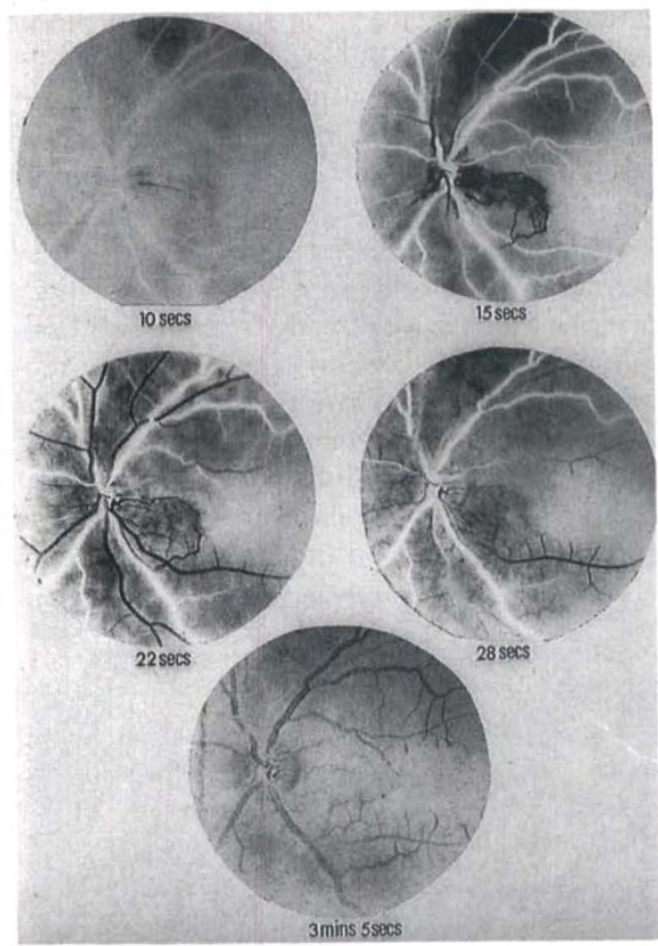

Fig. 2b.

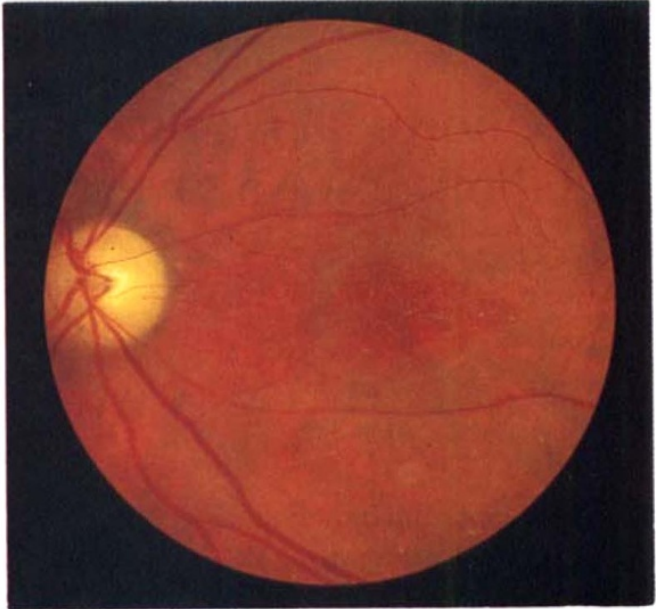

Fig. 2c.

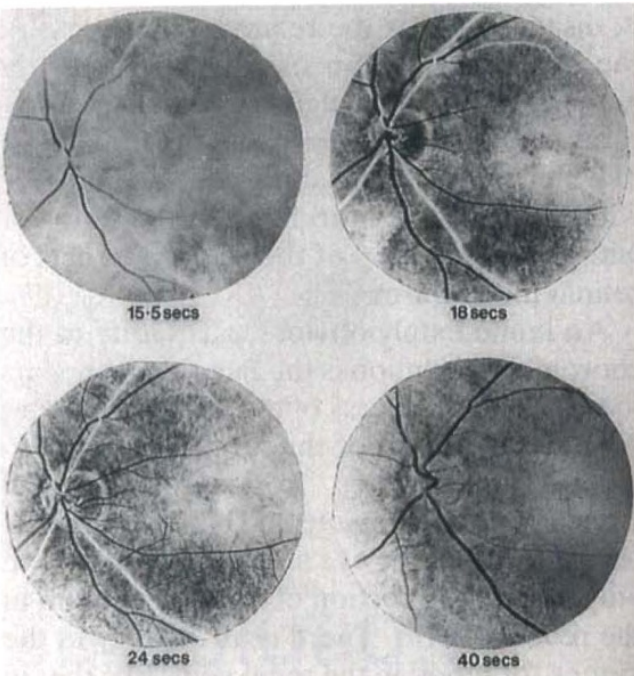

Fig. 2d.

Fig. 2. (a) Fundus photograph and

(b) Fluorescein angiogram of a patient suffering temporary occlusion of the central retinal artery following an orbital injury to show considerable dilatation of patent cilio-retinal vessels

(c) Fundus photograph and

(d) Fluorescein angiogram after spontaneous restoration of retinal circulation to show return of cilio-retinal system to its normal undilated state.

(Case previously published by Chawla J, Trans Ophthalmol Soc UK 1972; 92: 777-84) 


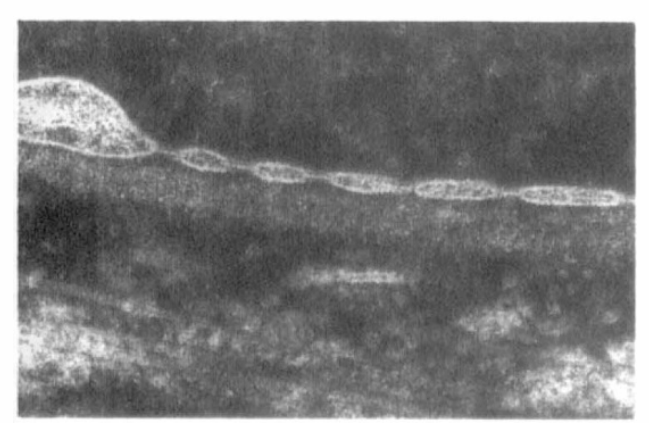

Fig. 3. Transmission electronmicroscope picture of the chorio-capillaris to show capillary fenestrations.

many years ago showed that choroidal circulation could be maintained relatively constant over a wide range of perfusion pressures achieved by alteration of IOP and of systemic blood pressure.

The choroidal circulation is exquisitely sensitive to carbon dioxide tension in the circulating blood and probably also to the acid products of metabolism, all of which cause choroidal vasodilatation and increased choroidal blood flow. This is well exemplified in a patient with a temporary obstruction of the central retinal artery who showed a gross dilatation of a cilio-retinal artery while the retina was ischaemic but whose cilio-retinal system returned to normal dimensions when blood flow was re-established in the central retinal artery (Fig. 2).

Although there must be good reasons as to why we need such a high blood flow in the choroid it is interesting that not only does it seem surplus to retinal metabolic requirements but additionally the retina unlike the brain can withstand quite prolonged periods of total ischaemia being capable of surprising morphological and functional recovery after periods of total ischaemia lasting up to one hour. ${ }^{5}$ This prompts us to ask why we need such a large flow of blood in the choroid. Is it to cool the retina by removing heat generated during visual transduction? Is it, as recently suggested by Damato in my Department, a mechanism to keep the retina warm and functioning by preventing the depression of temperature which might be associated with the exposed position of the eye in relation to the external environment? Returning to the analogy of the kidney where a large blood flow may be needed in relation to the mechanisms involved in glomerular filtration and tubular re-absorption, is the high choroidal blood flow related to fluid exchange across the posterior blood ocular barrier and, particularly, to the large outward movement of fluid which has been shown to exist from the vitreous cavity to the choroid? ${ }^{6}$ Lastly, is the high flow related to the relative separation of the outer retina from its blood supply and the need to maintain a high concentration gradient of required metabolites (and a low concentration of waste products of metabolism) to aid diffusional exchange?

A striking feature of the choriocapillaris is the presence of fenestrations which have been thought to be necessary to aid the supply of metabolites to the retina(Fig. 3). Many highly metabolising tissues, however, do not need such a structural modification in their capillary walls. We know that the capillaries of the choroid, unlike those of the retina, readily leak plasma protein as demonstrated on fluorescein angiography. Plasma proteins are excluded from the eye in the interests of optical clarity and the leakiness of the choroidal capillaries demands the presence of a diffusion barrier between the choroid and the retina. It seems likely that the fenestrations in the choroidal capillaries are needed to allow egress of plasma albumen and, more particularly, retinol binding protein (RBP) so that the all-important vitamen A can reach the retina. An important role of the retinal pigment epithelium (RPE) is therefore selectively to transfer retinol from its carrier protein and arrange for its transfer to the retina while at the same time excluding from the interior of the eye plasma albumin and proteins of a size similar to RBP which would otherwise cloud vision.

To reach the retina metabolites from the choriocapillaris must pass progressively through Bruch's membrane, the RPE and the interphotoreceptor space (IPS). Of these the RPE is obviously of the most crucial importance. There is now ample evidence that the RPE actively and selectively transfers molecules to and from the retina and, importantly, is involved in the continuous outward movement from the vitreous cavity and subretinal space of water and other molecules 


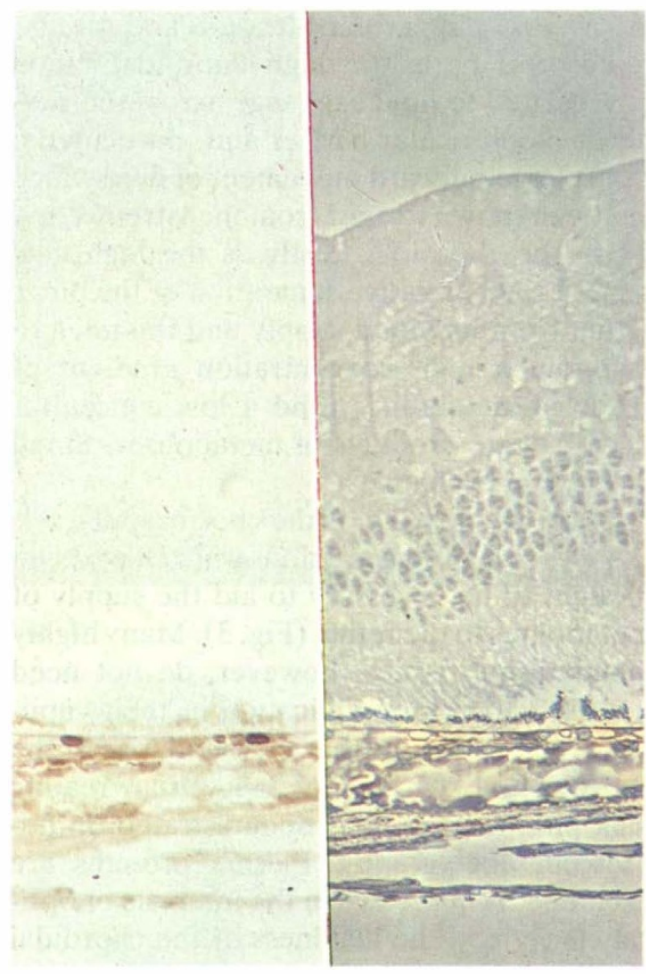

Fig. 4a \& 4b.

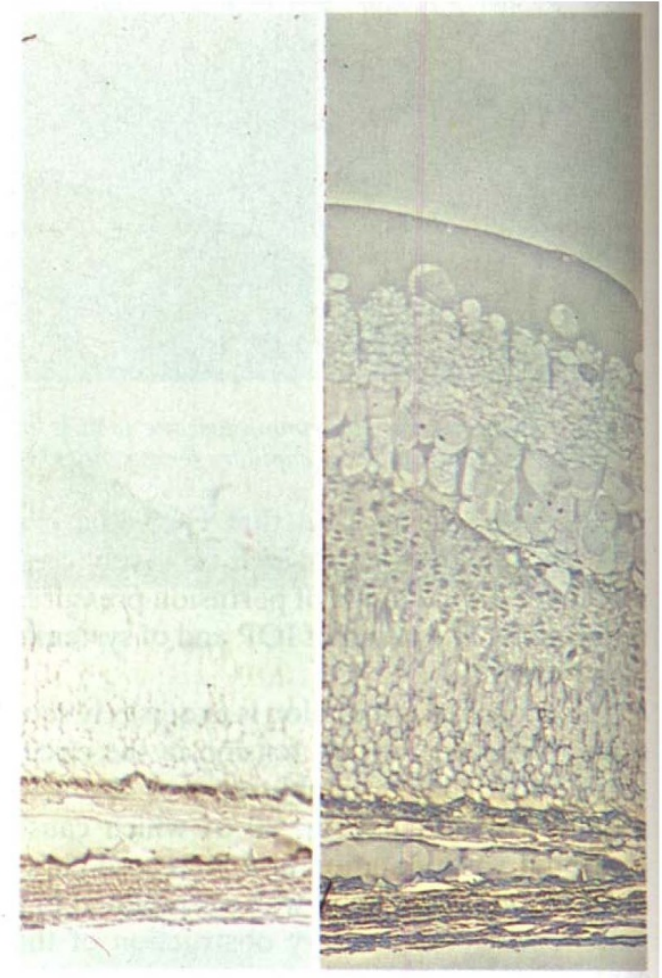

Fig. 4c \& 4d.

Fig. 4. Breakdown in the posterior blood ocular barrier resulting from poisoning by various doses of sodium iodate and demonstrated by the diffusion of intravenously injected horseradish peroxidase through the whole thickness of the retina via the damaged RPE (experiments carried out in rabbit by McKechnie and Foulds (unpublished)).

$a \& c$, control tissue $b \& d$, effects of intravenous sodium iodate.

which, if allowed to accumulate, would lead to retinal separation. (See Steinberg for review) ${ }^{7}$

Ionic movement across the RPE apical and basal membranes is reflected in the standing potential of the eye and the 'c' wave of the ERG, both of which can be increased in amplitude by the administration of substances which stimulate ionic transfer such as sodium azide, ${ }^{8,9}$ or reduced or even abolished by poisons which selectively inhibit RPE function, notably sodium iodate as demonstrated many years ago by Noell. ${ }^{10}$

Not unexpectedly, poisoning of the RPE by sodium iodate leads to severe metabolic upset and structural change in the related outer retina and to a breakdown in the posterior blood ocular barrier as demonstrated by the use of tracers such as horseradish peroxidase (HRP) (Fig. 4). If the transfer of water from the potential subretinal space to the choroid is mediated by active work on the part of the RPE one might expect that poisoning the RPE would reduce this outward movement of water. Surprisingly the reverse seems to be the case, poisoning by sodium iodate leading to an increased outward movement of water rather than a decrease as shown by Marmor using his localised retinal detachment technique $^{11}$ and by ourselves using tritiated water as a tracer. One has to postulate that the outward movement of water from the subretinal space to the choroid is only partly dependent on active transport by the RPE and that other physical factors not requiring a functionally intact RPE are also at work. These factors 
may include the colloid osmotic pressure of protein in the extravascular tissue spaces of the choroid as originally suggested by Bill. ${ }^{12}$

Recently the subretinal space itself has become an area of great interest and, particularly, the interphotoreceptor matrix which fills it. This matrix is formed of a mixture of complex glycoproteins and glycosaminoglycans and contains an important transport protein for Vitamin A (interphotoreceptor matrix retinol binding protein, IRBP). The matrix is structurally inhomogeneous being highly glycated in the vicinity of cone outer segments. ${ }^{13}$ The matrix may have adhesive properties important in retinal apposition. ${ }^{7}$ It obviously interposes a diffusion barrier between the choroid and the retina and at present its role in retinal metabolism remains conjectural. The highly polymerised glycosaminoglycans and glycoproteins of the matrix, however, are water retentive so maintain an interval between the apical RPE and the outer retina perhaps in this way helping to avoid unwanted local concentrations of metabolites or waste products. $^{14}$

An essential aspect of retinal metabolism is, of course, that related to the synthesis and phagocytic destruction of outer segments; processes that require a large turnover of phospholipid for plasma membrane synthesis and of retinal for the synthesis of visual pigment. There is evidence that some components of the matrix may be important in this process through their inhibitory effect on phagocytosis of receptor outer segments (ROS) by the RPE. ${ }^{15}$

The supply of lipid needed for the continuous synthesis of ROS plasma membrane has to take place not only through the RPE but across Bruch's membrane which in health does not appear to provide any barrier to the movement of even large molecules. In the elderly, however, there may be an accumulation of lipid containing waste products of phagocytosis in Bruch's membrane and these may impede the outward movement of water from the RPE to the choroid and be of relevance to the genesis of RPE detachment as suggested on theoretical grounds by Damato in $1985^{16}$ and as demonstrated by Bird and Marshall. ${ }^{17}$ It is interesting that although the effects of increased resistance in Bruch's membrane to the outward movement of molecules from the RPE to the chorotid has received a great deal of attention, little has been said about the effect changes in the permeability of Bruch's membrane may have on the inward diffusion of metabolites from the choroid to the retina. It may be that normally such metabolites are delivered to the RPE in excess and that even a moderate reduction in supply to the RPE related to increased resistance in Bruch's membrane might not influence the ability of the RPE to transfer sufficient to the retina to meet its metabolic requirements.

The whole area of metabolic exchange across the posterior blood ocular barrier to the retina is currently of great interest, not only because of an increased awareness of many choroidal vascular diseases and disorders of the retinal pigment epithelium which may affect it but also in relation to our understanding of the mechanism of action of photocoagulation in such conditions as diabetic retinal neovascularisation. Undoubtedly laser energy delivered in panretinal photocoagulation is absorbed at the level of the RPE and choroid and in ways which we do not yet understand leads to metabolic changes in the retina which are associated with regression of neovascular complexes at a distance from the area treated. Whether these effects are the result of altered blood flow and oxygenation in the choroid and retina or mediated through the release of metabolically active molecules from damaged RPE is a subject of intense debate.

The scanning and transmission electron micrographs were prepared by Mrs. Dorothy Aitken and the fluorescein angiograms by Mrs. Anne Currie to both of whom I am grateful.

\footnotetext{
References

${ }^{1}$ Alm A and Bill A. Ocular and optic nerve blood flow at normal and increased intraocular pressure in monkeys (Macaca Irus) a study with radioactively labelled microspheres including flow determinations in brain and other tissues. Exp Eye Res 1973, 15: 15 .

${ }^{2}$ Wilson TM, Strang R, Wallace J, Horton PW, Johnston NF. The measurement of the choroidal blood flow in the rabbit using Krypton 85. Exp Eye Res 1973, 16: 421-55.
} 
${ }^{3}$ Hayreh SS. The segmental nature of the choroidal vasculature. Br J Ophthalmol 1975, 59: 631-48.

${ }^{4}$ Wilson TM, MacKenzie ET, Strang R. Factors influencing ocular blood flow. In: Vision Circulation, Cant JS (Ed) St Louis 1976. The C V Mosby Co pp 45-49.

${ }^{5}$ Johnson NF. Electronmicroscopy of acute retinal ischaemia in the rabbit and a study of the pattern of recovery. In: Vision and Circulation, JS Cant (Ed) St Louis. The C V Mosby Co 1976 pp 79-92.

${ }^{6}$ Moseley H, Foulds WS, Allan D, Kyle PM. Routes of clearance of radioactive water from the rabbit vitreous. Br J Ophthalmol 1984, 68: 145-51.

${ }^{7}$ Steinberg RH. Research update: report from a workshop on cell biology of retinal detachment. Exp Eye Res 1986, 43: 696-706.

${ }^{8}$ Noell WK. Azide sensitive potential differences across the eye bulb. Am J Physiol 1952, 170: 217-38.

${ }^{9}$ Foulds WS and Ikeda $\mathrm{H}$. The effects of retinal detachment on the induced and resting ocular potentials in the rabbit. Invest Ophthalmol Vis Sci 1966, 5: 93-108.

${ }^{10}$ Noell WK. Studies on the electrophysiology and the metabolism of the retina. USAF School of Aviation Medicine. Project No. 21/1201/0004. Report No. 1, 1221953.
${ }^{11}$ Marmor MF, Abdul Rahim AS, Cohen DS. The effect of matabolic inhibitors on retinal adhesion and subretinal fluid absorption. Invest Ophthalmol Vis Sci 1980; 19: 893-903.

${ }^{12}$ Bill A. Some aspects of tissue fluid dynamics in the eye. In: Vision and Circulation, Cant JS (Ed) St Louis 1976. The C V Mosby Co 333-8.

${ }^{13}$ Johnson LV and Hageman GS. Enzymatic characterisation of peanut agglutinin binding components in the retinal interphotoreceptor matrix. Exp Eye Res 1987, 44: 553-65.

${ }^{14}$ Foulds WS. Is your vitreous really necessary? The role of the vitreous in the eye with particular reference to retinal attachment, detachment and the mode of action of vitreous substitutes. Eye 1987, 1: 641-64.

${ }^{15}$ Gregory CY, Converse CA, Foulds WS. Effect of glycoconjugates on rod outer segment phagocytosis by retinal pigment epithelial explants in vitro assessed by a specific double radioimmunoassay procedure. Current Eye Rés. (In Press).

${ }^{16}$ Damato BE. The subretinal stimulus to neovascularisation in Mackenzie symposium on retinal neovascularisation. Glasgow 1985 (unpublished).

${ }^{17}$ Bird AC and Marshall J. Retinal pigment epithelial detachments in the elderly. Trans Ophthalmol Soc UK 1986, 105: 674-820. 